\title{
A caracterização da produção bibliográfica nas práticas hospitalares em terapia ocupacional no Brasil: uma revisão da literatura de 1990 a 2007*
}

\author{
The characterization of the literature production \\ in occupational therapy hospital practice in Brazil: \\ a literature review from 1990 to 2007
}

\author{
Sandra Maria Galheigo ${ }^{1}$, Juliana Russo Antunes ${ }^{2}$
}

GALHEIGO, S. M.; ANTUNES, J. R. A caracterização da produção bibliográfica nas práticas hospitalares em terapia ocupacional no Brasil: uma revisão da literatura de 1990 a 2007. Rev. Ter. Ocup. Univ. São Paulo, v. 19, n. 2, p. 91-99, maio/ago. 2008.

RESUMO: Este artigo se propõe a apresentar a caracterização da produção do conhecimento referente às práticas hospitalares em terapia ocupacional, através de estudo da produção bibliográfica, de 1990 a 2007, de terapeutas ocupacionais com inserção profissional no Brasil. Foi analisada a produção na forma de artigos de periódicos, livros, capítulos de livro e resumos ampliados dos Anais de três Congressos Brasileiros de Terapia Ocupacional, o que resultou num levantamento bibliográfico de 116 registros. O material foi organizado e analisado por período, região de inserção profissional do autor, tipo de publicação, periódicos e indexação, tipo de produção (pesquisa, revisão bibliográfica, relato de experiência, trabalhos de natureza teórica, metodológica e técnica) e domínios de publicação. Os resultados apontam para uma crescente produção nos últimos 8 anos, predominantemente de terapeutas ocupacionais inseridos profissionalmente no sudeste. Os artigos são o tipo de publicação mais comum dos quais a metade é em periódicos indexados e a outra em não indexados. Os resumos ampliados de congressos começam a ser uma forma importante de produção, seguidos dos capítulos de livro. O relato de experiência é o tipo de produção mais realizada e a pesquisa começa a despontar depois de um crescimento substancial nos últimos 3 anos. Para a área se consolidar e qualificar, fazendo com que conhecimento seja divulgado e compartilhado é essencial que terapeutas ocupacionais continuem investindo na produção, dando continuidade e potencializando a tendência crescente que se verifica nesse estudo.

DESCRITORES: Terapia ocupacional/tendências. Processo saúde-doença. Assistência hospitalar. Serviço hospitalar de terapia ocupacional. Indicadores de produção científica.

\footnotetext{
* Artigo produto de projeto de pesquisa Práticas hospitalares em terapia ocupacional: um estudo sobre a consolidação do campo no Estado de São Paulo, que a pesquisadora vem desenvolvendo com o apoio do CNPq.

1 Professora doutora, docente do Departamento de Fisioterapia, Fonoaudiologia e Terapia Ocupacional da Faculdade de Medicina da USP.

2 Bolsista de Iniciação Científica - PIBIC.

Endereço para correspondência: Centro de Docência e Pesquisa em terapia Ocupacional da FMUSP, Rua Cipotânea, 51 - Cidade Universitária, 05508-900, São Paulo-SP.
} 


\section{INTRODUÇÃO}

A atuação e publicação da terapia ocupacional na produção do cuidado em saúde, em especial das pessoas sob cuidados clínicos e cirúrgicos em atendimento hospitalar, ambulatorial ou domiciliar, tem se ampliado nas últimas décadas. Esta constatação pode ser verificada a partir da análise da produção bibliográfica que traz subsídios para a compreensão da constituição da área, apontando características e tendências da construção de seus saberes e práticas. A análise de produção científica no período de 1990 a 2006, revelou que os domínios da terapia ocupacional em práticas hospitalares em que se têm mais publicado são o de atenção à gestante, puérpera e neonato; à criança e adolescente em enfermarias pediátricas; ao adulto e idoso em hospital geral; a pessoas com câncer e/ou HIV/ AIDs e; em menor medida, o de fundamentos históricos, filosóficos e metodológicos do campo (GALHEIGO, 2007).

Artigos de pesquisa, trabalhos de natureza teórica, metodológica e técnica, relatos de experiência e revisão da bibliografia refletem determinada realidade nacional e regional e mostram tendências e características que permitem compreender as potencialidades e limitações das chamadas práticas hospitalares assim como de sua produção bibliográfica. Assim, este artigo se propõe a apresentar uma caracterização da produção científica das práticas hospitalares em terapia ocupacional, de 1990 a 2007, por tipos de veículos de divulgação e sua indexação, procedência dos autores, regionalização do trabalho e tipos de produção (artigo de pesquisa, relato de experiência, revisão de bibliografia e trabalho de caráter teórico, metodológico ou técnico).

\section{Sobre os procedimentos metodológicos}

Para a realização desse trabalho, foi feito um levantamento bibliográfico compreensivo da produção da terapia ocupacional brasileira em práticas hospitalares, referente ao período de 1990 a 2007. Foram analisados artigos em periódicos indexados e não indexados, livros, capítulos de livro e resumos ampliados dos Anais dos Congressos Brasileiros de Terapia Ocupacional.

Foram consultadas as bases de dados LILACS, MEDLINE e SCIELO, usando-se o descritor serviço hospitalar de terapia ocupacional e combinações dos descritores terapia ocupacional, hospitalização e assistência hospitalar. Outras combinações com o descritor terapia ocupacional foram procuradas, e na medida em que novos descritores eram identificados nos textos, eles passavam a incorporar o repertório de busca. Ao final foram utilizados em combinação com terapia ocupacional os seguintes descritores: criança hospitalizada, adolescente hospitalizado, neoplasias, doenças reumáticas, unidade de terapia intensiva neonatal, alojamento conjunto, ginecologia, neonatologia, pediatria, ortopedia, neurologia, oncologia, nefrologia, geriatria, clínica médica, clínica cirúrgica e unidade de queimados.

No caso dos periódicos Cadernos de Terapia Ocupacional da UFSCar e Revista Prática Hospitalar, que não têm mecanismos automáticos de busca, foi feita varredura manual. Foram realizadas buscas complementares a partir das referências das produções previamente identificadas e de consulta ao Curriculum Lattes dos autores previamente identificados. Tal consulta permitiu a identificação de produções em livros e outros periódicos não indexados. Possibilitou também a identificação de um artigo em periódico internacional, que foi incluído no corpus de análise por ter co-autoria brasileira e versar claramente sobre a atenção à população sobre cuidados clínicos e cirúrgicos no Brasil.

O corpus gerado pelo levantamento bibliográfico consistiu de 116 registros de produções, cujos dados foram organizados em um banco de dados, analisado sob diferentes recortes: tipos de veículos de divulgação e sua indexação, autoria e procedência da inserção profissional dos autores, regionalização do trabalho e tipos de produção (artigo de pesquisa, relato de experiência, revisão de bibliografia e trabalho de caráter teórico, metodológico ou técnico).

\section{A produção por volume, período e tipo de publi- cação}

A produção referente às práticas hospitalares em terapia ocupacional é relativamente recente já que $80 \%$ de seu total é do período compreendido entre os anos de 2000 a 2007 . O corpus gerado pelo levantamento bibliográfico consta de 116 registros e é composto por 24 artigos em periódicos indexados e 24 em periódicos não indexados, 25 capítulos de livros, 39 resumos ampliados e 4 livros de terapia ocupacional (Tabela 1). Analisando a produção por qüinqüênios, constamos um crescimento importante, já que respectivamente eles apresentam 12, 11, $32 \mathrm{e} 61$. Entretanto, recentemente os resumos ampliados têm tido um importante impacto nessa variação, já que sem eles os qüinqüênios passariam a contar respectivamente com 12, 6, 31 e 28 produções. Importante apontar que o último qüinqüênio está incompleto, já que a produção foi levantada até 2007 , correspondendo a $60 \%$ do tempo total previsto (Figura 1). 
GAlHeigO, S. M. et al. A caracterização da produção. Rev. Ter. Ocup. Univ. São Paulo, v. 19, n. 2, p. 91-99, maio/ago. 2008.

Tabela 1 - Produção por período e tipo de publicação - 1990-2007

\begin{tabular}{|c|c|c|c|c|c|c|c|c|c|}
\hline \multirow{2}{*}{$\begin{array}{c}\begin{array}{c}\text { Tipo de } \\
\text { produção }\end{array} \\
\text { Período }\end{array}$} & \multirow{2}{*}{$\begin{array}{c}\text { Artigos } \\
\text { (periódicos } \\
\text { indexados) }\end{array}$} & \multirow{2}{*}{$\begin{array}{c}\text { Artigos } \\
\text { (periódicos não } \\
\text { indexados) }\end{array}$} & \multirow{2}{*}{ Livros } & \multirow{2}{*}{$\begin{array}{c}\text { Capítulos } \\
\text { de livros }\end{array}$} & \multicolumn{4}{|c|}{ Resumos ampliados } & \multirow{2}{*}{ Total } \\
\hline & & & & & $\begin{array}{c}\text { CBTO } \\
1997 \\
\end{array}$ & $\begin{array}{c}\text { CBRO } \\
2005 \\
\end{array}$ & $\begin{array}{c}\text { CBTO } \\
2007 \\
\end{array}$ & Outros & \\
\hline $1990-4$ & 3 & & 1 & 8 & & & & & 12 \\
\hline $1995-9$ & 2 & 4 & & & 5 & & & & 11 \\
\hline $2000-4$ & 9 & 10 & 2 & 10 & & & & 1 & 32 \\
\hline 2005-7 & 10 & 10 & 1 & 7 & & 20 & 13 & & 61 \\
\hline Total & 24 & 24 & 4 & 25 & & 3 & & & 116 \\
\hline
\end{tabular}

Figura 1 - Produção total e sem resumos ampliados, por período - 1990-2007

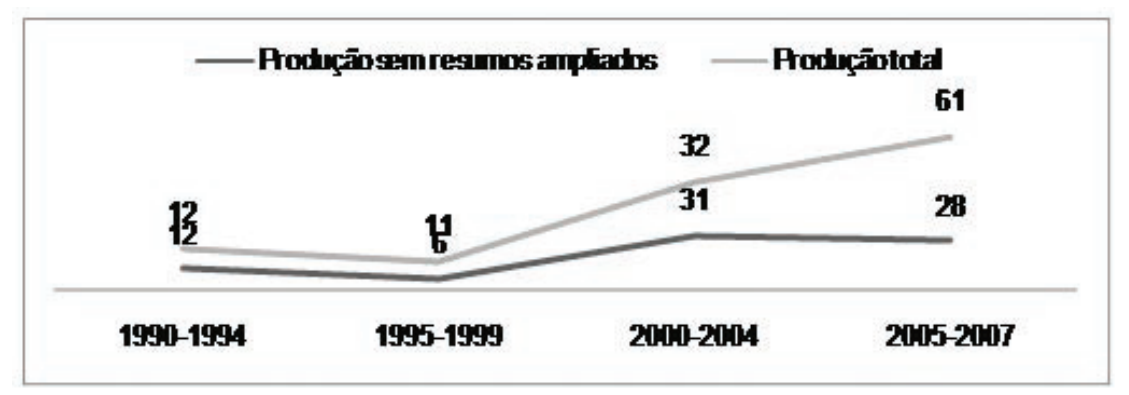

Foram analisadas as produções de três Congressos Brasileiros de Terapia Ocupacional, a saber: o V Congresso Brasileiro \& IV Simpósio Latino-Americano de Terapia Ocupacional (1997) e os IX e X Congressos Brasileiros de Terapia Ocupacional (2005 e 2007). A análise de apenas três eventos em 28 anos deve-se ao fato da publicação dos resumos ampliados dos CBTOs ser prática recente. Apenas os dois últimos eventos, em 2005 e 2007, abriram a possibilidade de publicação de resumo ampliado de todas as apresentações (mesas-redondas, conferências e temas livres). O evento de 1997 publicou anais de resumos ampliados apenas das produções dos convidados e não dos temas livres, o que totalizou apenas cinco produções sobre práticas hospitalares. Esse número já cresce significativamente nos CBTO/2005 e CBTO/2007 com respectivamente 20 e 13 produções na área. Apenas um resumo ampliado não é proveniente de CBTO e foi incluído por ter sido publicado em periódico indexado.

As produções de três dos livros foram também contabilizadas por seus capítulos (após seleção daqueles que têm correlação direta com a área estudada). Com relação aos quatro livros é importante assinalar que um deles é resultante da publicação de uma dissertação de mestrado (SERVANTES, 2002) e outro é um compêndio com capítulos sobre os vários campos da terapia ocupacional, dos quais cinco foram identificados como da área estudada (CAVALCANTI; GALVÃO, 2007). Dois desses livros apresentam um foco maior em práticas hospitalares e são constituídos por capítulos de vários autores. Entretanto, nem todos os seus capítulos foram categorizados como de práticas hospitalares em terapia ocupacional, já que um livro inclui capítulos sobre Fisioterapia e Fonoaudiologia (KUDO et al., 1994) e o outro apresenta capítulos em reabilitação física (De CARLO; LUZO, 2004). Nesse caso optou-se por considerar os capítulos que em seu contexto explicitassem alguma prática ou reflexão próprias do contexto hospitalar e da terapia ocupacional, o que resultou na identificação de oito capítulos no primeiro e nove no segundo. Três capítulos de livro identificados como relativos às práticas hospitalares da terapia ocupacional foram publicados em livros interdisciplinares sobre temáticas específicas, tais como o transplante de medula, humanização e atendimento 
em hospital pediátrico (MASTROPIETRO et al., 2003; MITRE, 2006; MARIA; KUDO, 2006).

Os artigos são o tipo de publicação mais freqüente em práticas hospitalares, totalizando 48 produtos. Se considerarmos que o montante de produção sobre a área no período anterior a 1990 (identificada por essa pesquisa) era de apenas dois artigos em periódicos, constata-se que houve um significativo incremento da produção. Esta segue a tendência da produção geral, tendo $80 \%$ dos artigos sido produzidos nos últimos oito anos (Tabela 1).

No que se refere à indexação, metade dos 48 artigos foi publicada em periódicos indexados em base de dados e a outra metade em não indexados. Os periódicos indexados com mais artigos relativos às práticas hospitalares em terapia ocupacional é a Revista de Terapia Ocupacional da USP, totalizando cinco registros, seguida do periódico Pediatria (São Paulo) com três registros. As demais produções ficam pulverizadas em 13 periódicos. Apenas um artigo foi publicado em periódico internacional de terapia ocupacional com impacto avaliado pelo Institute for Scientific Information (ISI), cinco artigos foram publicados em três periódicos indexados na Base SCIELO e todos os demais na base LILACS. Em relação aos periódicos não indexados, nove dos 24 artigos foram publicados nos Cadernos de Terapia Ocupacional da UFSCar, seis na Revista Prática Hospitalar e quatro na Revista do Centro de Estudos de Terapia Ocupacional (CETO). As demais produções estão distribuídas por quatro outras revistas, duas das quais foram edições especiais com volume único (Tabela 2).

Tabela 2 - Produção por periódico e base de indexação - 1990-2007

\begin{tabular}{|c|c|c|}
\hline Periódicos Indexados & Bases de Dados & Total \\
\hline American Journal of Occupational Therapy* & MEDLINE; CINAHL & 1 \\
\hline Ciência \& Saúde Coletiva & $\begin{array}{l}\text { SCIELO; LILACS;SCOPUS; LATINDEX; INDEX MEDICUS LATINO } \\
\text { AMERICANO }\end{array}$ & 2 \\
\hline Folha Médica & $\begin{array}{l}\text { BIOL. ABSTRACTS; EXCERPTA MEDICA; INDEX MEDICUS } \\
\text { LATINO AMERICANO }\end{array}$ & 1 \\
\hline Medicina (Ribeirão Preto) & LILACS; SCOPUS; EXCERPTA MEDICA & 2 \\
\hline Mundo da Saúde & LILACS & 1 \\
\hline Pediatria (São Paulo) & LILACS & 3 \\
\hline Pediatria Moderna & $\begin{array}{l}\text { LILACS; BIOL. ABSTRACTS; INDEX MEDICUS LATINO AMERI- } \\
\text { CANO }\end{array}$ & 1 \\
\hline Revista Brasileira de Cancerologia & LILACS; INDEX MEDICUS LATINO AMERICANO & 1 \\
\hline Revista Brasileira de Reumatologia & $\begin{array}{l}\text { SCIELO; SCOPUS; LILACS; EXCERPTA MEDICA; INDEX MEDI- } \\
\text { CUS LATINO AMERICANO }\end{array}$ & 2 \\
\hline Revista da Spagesp & LILACS & 1 \\
\hline Revista de Terapia Ocupacional da USP & LILACS & 5 \\
\hline Revista Diagnóstico e Tratamento & LILACS & 1 \\
\hline Revista Paulista de Pediatria & SCIELO; LILACS; INDEX MEDICUS LATINO AMERICANO & 1 \\
\hline Revista Pediatria Moderna & $\begin{array}{l}\text { LILACS; BIOLOGICAL ABSTRACTS; INDEX MEDICUS LATINO } \\
\text { AMERICANO }\end{array}$ & 1 \\
\hline Temas sobre desenvolvimento, & LILACS & 1 \\
\hline Total & & 24 \\
\hline \multicolumn{3}{|l|}{ Periódicos Não Indexados } \\
\hline Cadernos - Centro Universitário São Camilo & & 2 \\
\hline Cadernos de Terapia Ocupacional da UFSCAR & & 9 \\
\hline Revista de Terapia Ocupacional da Bahiana & & 1 \\
\hline Revista de Terapia Ocupacional PUCCAMP & & 1 \\
\hline Revista do Centro de Estudos de Terapia Ocupacional & & 4 \\
\hline Revista Dor (São Paulo) & & 1 \\
\hline Revista Prática Hospitalar & & 6 \\
\hline Total & & 24 \\
\hline
\end{tabular}


Em suma, o cenário da produção apresentado aponta para um recente aumento da produção da área e para uma tendência de produção de artigos maior do que dos demais tipos de publicação. Entretanto, a metade desses é realizada em periódicos não indexados e apenas um é em periódico internacional com fator de impacto avaliado. Interessante apontar que praticamente a metade dos artigos $(n=23)$ foi publicada em periódicos específicos de terapia ocupacional e a outra metade em periódicos da área da saúde em geral. Os capítulos de livro também são importantes fontes de transmissão de conhecimento da área e a publicação de resumos ampliados nos anais de Congresso mostra-se potencialmente importante para trazer vigor à divulgação de experiências e do conhecimento

\section{A produção por região e autoria}

Analisando a produção bibliográfica em práticas hospitalares por região geográfica do endereço profissional dos autores no momento da publicação, constata-se uma desigualdade acentuada. A região sudeste concentra 84,4\% da produção nacional $(n=98)$, seguida da região nordeste que detém $12 \%$ do total $(\mathrm{n}=14)$. As regiões norte, sul e centro-oeste contam juntas com $4,3 \%$ da produção total, tendo respectivamente 1,2 e 2 produções cada. Como não há estudos análogos sobre a produção científica da profissão no país, não é possível comparar padrões para se verificar se esse resultado expressa um padrão da profissão em geral ou se é uma particularidade da área.

$\mathrm{O}$ número de profissionais e de cursos de terapia ocupacional por região por si só não justifica a disparidade constatada. Dados sobre o número de profissionais por CREFITO em 2008, disponibilizados pelo Conselho Federal de Fisioterapia e Terapia Ocupacional, revelam que dos 11.228 profissionais do país, $53,51 \%$ estão localizados na região sudeste; $19,62 \%$ na nordeste; 13,55 $\%$ no sul e $13,32 \%$ nas regiões norte e centro-oeste. Há que se mencionar uma inconsistência no agrupamento regional no que refere ao Estado do Maranhão que faz parte do CREFITO 12, junto com estados da região norte e centro-oeste. Em relação à distribuição regional dos cursos de graduação em terapia ocupacional, dados de 2008 do Instituto de Pesquisas Educacionais Anísio Teixeira revelam que $60,3 \%$ dos cursos localizam-se na região sudeste, $17,4 \%$ na nordeste, $11,1 \%$ na região sul, $4,7 \%$ na região norte e $6,3 \%$ na centro-oeste (INEP 2008). Mesmo considerando que alguns cursos foram criados recentemente nas regiões norte e centro-oeste, é importante assinalar que a expansão tem se dado em âmbito nacional. Portanto, verificando tais dados nota-se que é a região sul que apresenta proporcionalmente menor produção quando comparada ao número de profissionais e cursos de terapia ocupacional existentes. Sem dúvida, há que se considerar que grandes complexos hospitalares estão no sudeste assim como os cursos de terapia ocupacional em universidades públicas e de programas de educação continuada (como aprimoramentos e residências profissionais). Assim, a pouca produção bibliográfica pode estar mais relacionada ao pouco incentivo à pesquisa e à produção do que à baixa inserção de terapeutas ocupacionais na área. Tais observações não têm objetivo de fazer qualquer forma de ranqueamento da produção por região, mas apontar as diferenças significantes. Acreditamos que compreender os motivos pelos quais acontecem tais diferenças regionais na produção pode auxiliar na identificação da necessidade de investimentos em termos das políticas de saúde, das políticas de educação permanente e inserção profissional e de incentivo à pesquisa em terapia ocupacional.

Quando a produção regional é analisada por período e região fica evidente que tanto as regiões sudeste e nordeste, respeitadas as devidas proporções, tiveram uma expansão importante no período de 2000 a 2007 (Figura 2). Entretanto, quando as publicações são analisadas por tipo, percebe-se que o período de 2005 a 2007 foi largamente influenciado pela publicação de anais de congresso mais compreensivos (Figura 3). Esse gráfico também demonstra que a região sudeste é que concentra a quase totalidade de artigos e capítulos de livro.

Analisando as produções por autoria, constatamos que os 116 produtos foram produzidos por 224 autores, não apenas terapeutas ocupacionais, já que há vários trabalhos interdisciplinares. A grande maioria desses autores $(\mathrm{n}=183)$ tem apenas um trabalho, e 21 têm duas produções cada. Isso aponta para a identificação de que há poucos autores que publicam regularmente em práticas hospitalares e mesmo assim o número máximo de produção por autor não ultrapassou o número de oito produtos. Identificando os terapeutas ocupacionais que mais têm publicado no assunto, chegou-se ao número de 16 profissionais dos quais oito têm 3 ou 4 produções e os outros oito têm de 5 a 8 trabalhos. Esses 16 profissionais trabalhavam na região sudeste no momento de sua publicação e apenas um não era do Estado de São Paulo; cinco eram docentes de cursos de terapia ocupacional, dos quais três de instituições públicas e dois de privadas. Sete eram terapeutas ocupacionais que no momento em que realizaram suas publicações trabalhavam em quatro hospitais de alta complexidade, dos quais dois são vinculados a uma mesma universidade com significante fomento à pesquisa. 
Figura 2 - Produção por região e período - 1990-2007

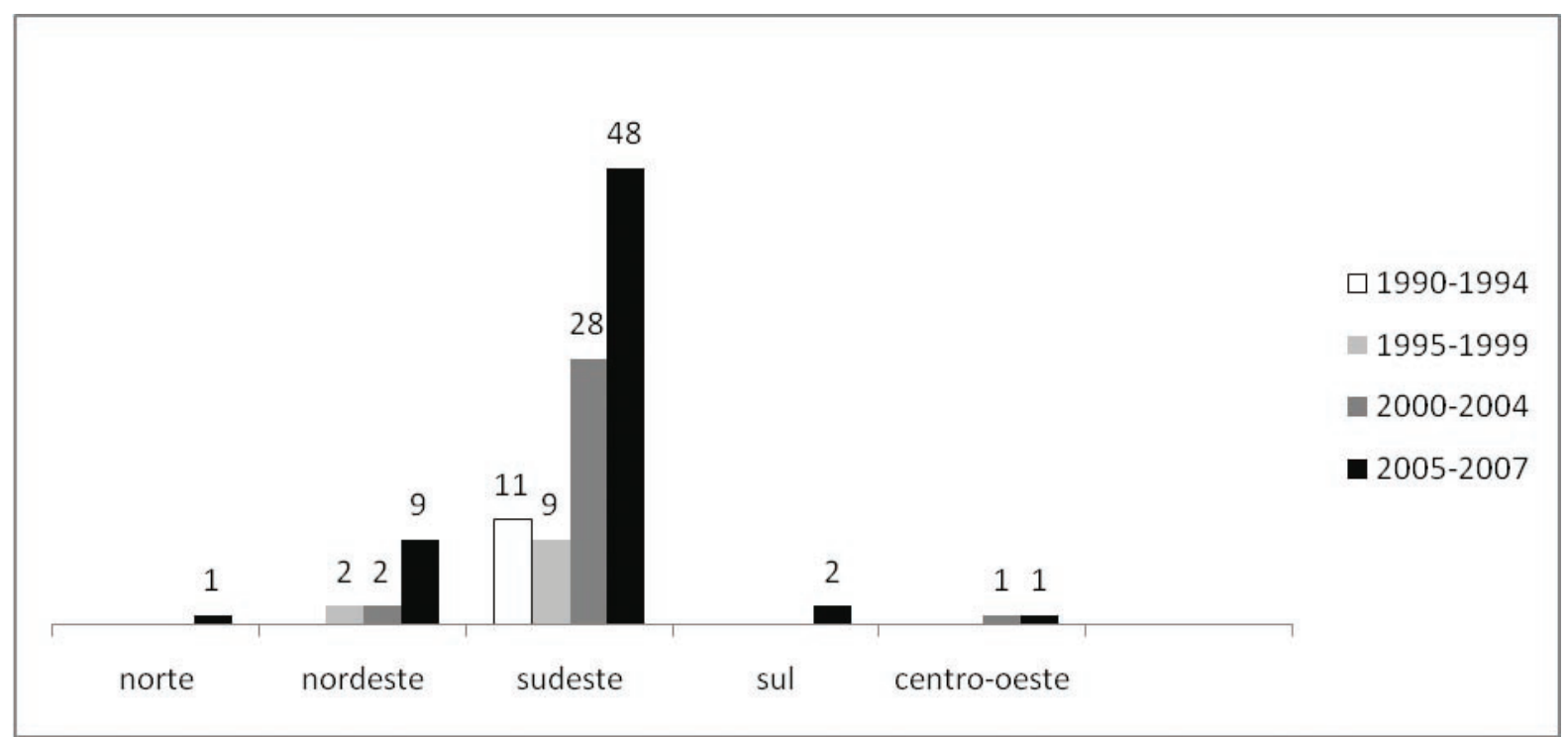

Obs. Uma das produções de autoria de profissionais trabalhando nas regiões NE e SE foi considerada duplamente nas respectivas regiões; os três livros (na forma de coletânea de capítulos de autores variados) foram excluídos para não gerar duplicidade.

Figura 3 - Produção por região e tipo de publicação - 1990-2007

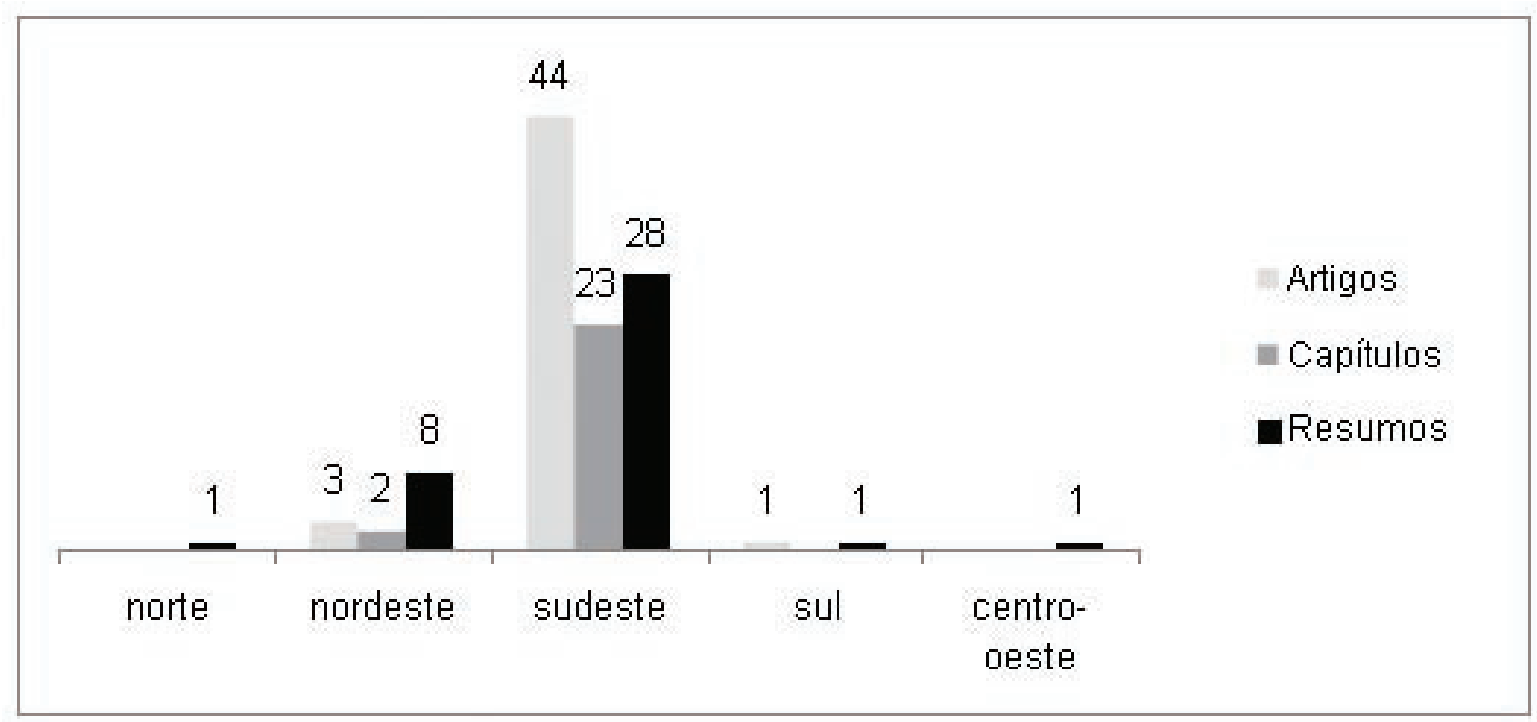

Em suma, os dados analisados por sua regionalização e autoria apontam para uma forte concentração da produção na região sudeste e em um pequeno grupo de profissionais cujas atividades principais são associadas à assistência. Trabalhar em hospital de alta complexidade e em instituições de ensino superior seja como docente, pesquisador ou 
profissional assistencial mostra ser um elemento facilitador para a produção.

\section{A produção por tipo de trabalho}

A produção em práticas hospitalares foi avaliada quanto ao tipo de trabalho que a originou, isto é, se foi resultado de pesquisa, revisão bibliográfica, relato de experiência ou se trata de trabalho de natureza teórica, metodológica e técnica, apresentado na forma de artigo ou de capítulo de livro de caráter didático ou de atualização. Quando algum trabalho podia se configurar em mais do que uma categoria, o critério de desempate foi a tendência prioritária identificada.

O relato de experiência é o tipo de produção mais realizado na área e tem tido crescimento constante ao longo do período estudado (Figura 4). Distribui-se de forma quase equitativa entre os artigos em periódicos indexados, aqueles não indexados e nos anais de congressos (Tabela 3 ). Essa constatação mostra a tendência que o terapeuta ocupacional tem de apresentar seu papel profissional através das contribuições que pode oferecer aos usuários de seus serviços e, portanto, das experiências que têm a contar. Os domínios em que os relatos de experiência mais acontecem é no trabalho com adultos e idosos, seguido do domínio de crianças e adolescentes. $\mathrm{O}$ trabalho da terapia ocupacional com neonatos e com pessoas com neoplasias também aparece com bastante freqüência. É interessante constatar que há uma crescente inclusão do discurso da humanização do cuidado na apresentação dos trabalhos (Figura 5).

Figura 4 - Produção por tipo e período - 1990-2007

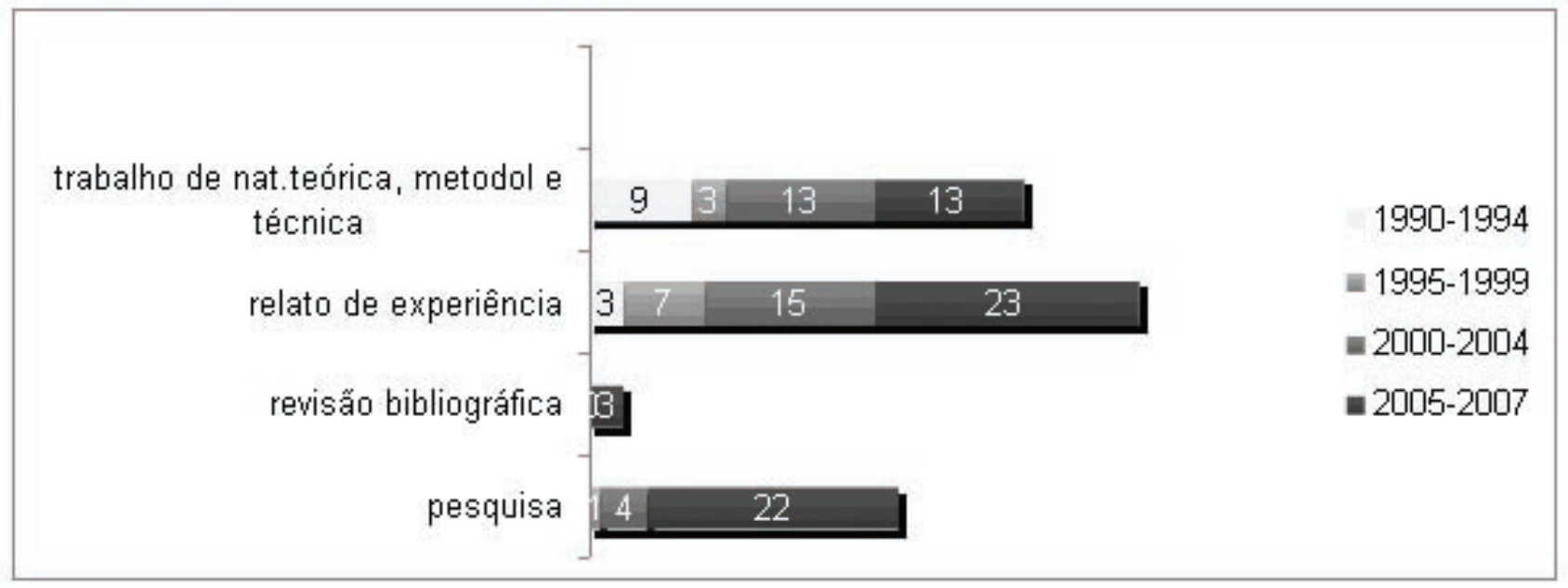

Tabela 3 - Produção por tipo de publicação e tipo de produção - 1990-2007

\begin{tabular}{l|c|c|c|c}
\hline & Pesquisa & $\begin{array}{c}\text { Revisão de } \\
\text { bibliografia }\end{array}$ & Relato de experiência & $\begin{array}{c}\text { Trabalho de natureza teórica, } \\
\text { metodológica e técnica }\end{array}$ \\
\hline $\begin{array}{l}\text { Artigo em periódico } \\
\text { indexado }\end{array}$ & 7 & 2 & 13 & 2 \\
\hline $\begin{array}{l}\text { Artigo em periódico não } \\
\text { indexado }\end{array}$ & 4 & 1 & 15 & 4 \\
\hline Livro & 1 & 0 & 0 & 3 \\
\hline Capítulo de livro & 0 & 0 & 2 & 23 \\
\hline Resumo ampliado & 15 & 0 & 18 & 6 \\
\hline Total & 27 & 3 & 48 & 38 \\
\hline
\end{tabular}


Figura 5 - Produção por tipo e domínio - 1990-2007

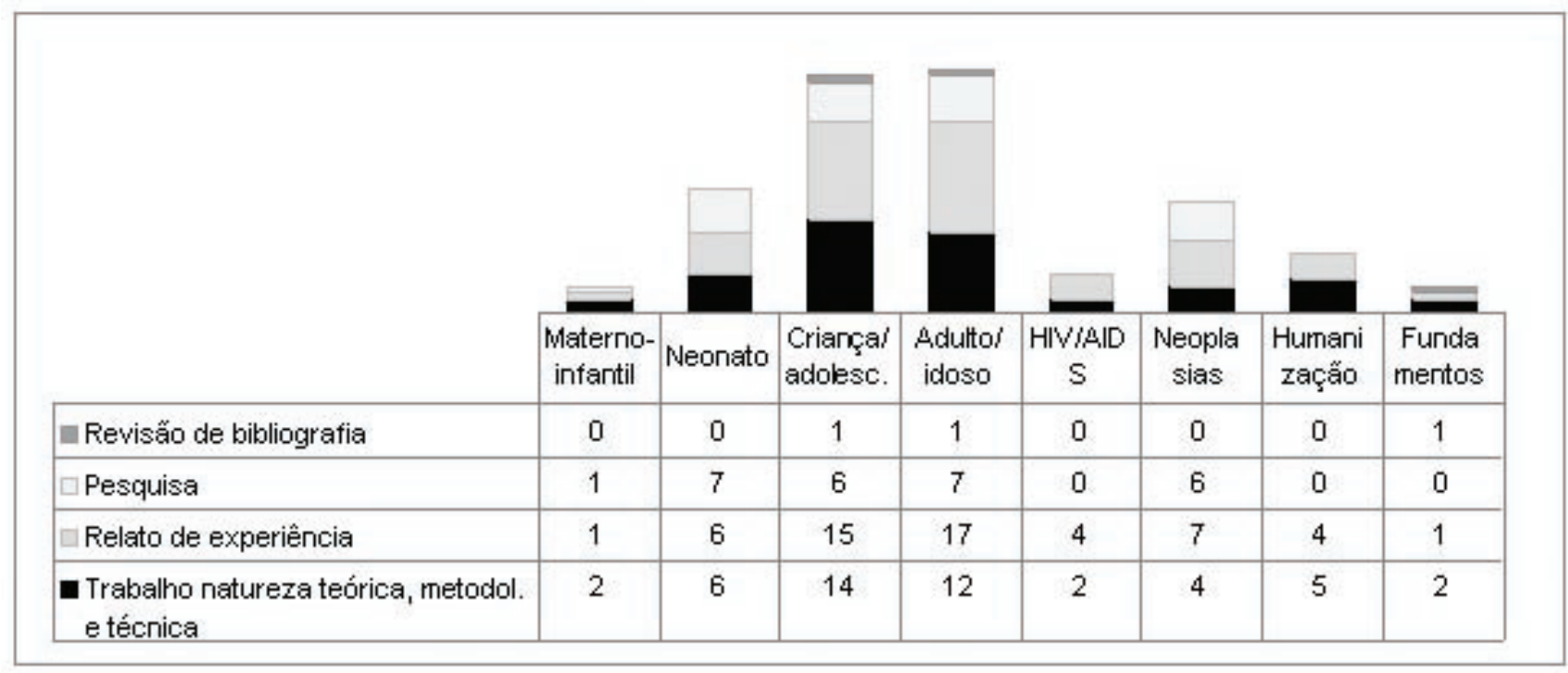

OBS.: As produções podem ter sido categorizadas em mais do que um domínio.

Os trabalhos de natureza teórica, metodológica e técnica são o segundo tipo de produção mais utilizado. Aparecem principalmente sob a forma de capítulos de livros e têm, em geral, finalidade didática e de atualização (Tabela 3). Seu padrão usual é o de configurar determinada realidade, contexto e necessidade de cuidado causada por agravos à saúde específicos e, a seguir, apresentar as contribuições da terapia ocupacional sob perspectiva teórica, metodológica e/ou técnica. Seu crescimento também tem sido constante ao longo do período estudado, mas ele de fato acompanha a publicação de livros (Figura 4; Tabela 3). Em relação aos domínios, constata-se que esse tipo de produção, por sua característica didática e de atualização, contempla os diferentes domínios, tendo o trabalho com crianças, adolescentes, adultos e idosos um destaque maior (Figura 5).

A produção gerada por pesquisa era praticamente nula até o ano 2000, e após 2005 passa a ter um crescimento significativo, que se manifesta, principalmente, nos anais de congresso e nos artigos em periódicos indexados (Figura 4). Esse crescimento pode estar associado com o aumento de profissionais cursando pós-graduação e o aumento de pesquisa nas universidades e deve ser uma tendência que esse crescimento continue acontecendo. Interessante notar que a pesquisa está mais presente prioritariamente no cuidado ao neonato, crianças, adolescentes, adultos e idosos e pessoas com neoplasias (Figura 5).

A revisão da bibliografia é o tipo de produção mais incipiente (Figura 4). Aparece em volume reduzido no domínio do cuidado a crianças, adultos e em fundamentos histórico-epistemológicos e é fruto dos últimos três anos (Figura 5, Tabela 3). Se considerarmos a intensa produção em saúde, é de se esperar que esse início seja uma nova tendência de produção da terapia ocupacional em práticas hospitalares.

\section{CONSIDERAÇÕES FINAIS}

A análise da produção bibliográfica em práticas hospitalares possibilitou a apreensão de um primeiro cenário do campo da produção do cuidado à saúde das pessoas em tratamento clínico ou cirúrgico.

Em linhas gerais, constatamos um sensível crescimento da produção do campo na última década, mas essa tendência precisa se consolidar e qualificar cada vez mais, se a terapia ocupacional pretende conquistar mais espaço no campo do cuidado em saúde nos hospitais, ambulatórios de seguimento e no domicílio, principalmente num cenário de disputa de mercado multiprofissional tão competitivo como esse. Portanto, é essencial que terapeutas ocupacionais consigam afirmar com clareza suas propostas e fundamentos teórico-metodológicos e se invistam da responsabilidade da produção de conhecimento que possa ser divulgado e compartilhado com os demais terapeutas ocupacionais assim como com membros da equipe multiprofissional.

Os desafios para os envolvidos com a assistência e o ensino da terapia ocupacional são o de ampliar o volume de pesquisas e publicações em periódicos indexados (que possuem regularidade e acesso via consulta em base de 
dados) e de garantir uma melhor distribuição regional da produção e do trabalho do terapeuta ocupacional. É importante também identificar-se domínios em que a atuação da terapia ocupacional pode ser potencializada.

A realização de pesquisas e a produção de conhecimento, contudo, tanto permitem compreender determinada realidade como contribuem para sua construção a partir de determinada perspectiva. Para se ter uma panorâmica menos enviesada é essencial que outras pesquisas de análise da produção sejam realizadas no sentido de aprofundar resultados e questões que foram trazidas por esse artigo como para confrontá-los ou detalhá-los em suas características regionais e locais e nos próprios domínios do campo.

GALHEIGO, S. M.; ANTUNES, J. R. The characterization of the literature production in occupational therapy hospital practice in Brazil: a literature review from 1990 to 2007. Rev. Ter. Ocup. Univ. São Paulo, v. 19, n. 2, p. 91-99, maio/ago. 2008.

\begin{abstract}
This paper intends to present the characterization of knowledge production regarding occupational therapy hospital practice in Brazil through literature review from 1990 to 2007. Papers, books, chapters of books and extended abstracts of three Brazilian Occupational Therapy Congresses were review, adding up to 116 items. Data were analyzed by publication domain, period of time, the author's professional address, type of publication, journals and their indexation, and type of production (research, literature review, experience report, theoretical, methodological or technical paper). The results show a crescent production for the last 8 years, produced by occupational therapists professionally located in the southeast. The papers are the commonest type of publication, being half of them published on indexed journals and the other half on non indexed ones. The extended abstracts have become an important means of production, followed by the book chapters. The experience report is the type of production which appears in larger numbers and research papers has increased in the last 3 years. For the area to consolidate and become qualified, making it easier to knowledge be divulged and shared, it is essential that occupational therapists carry on producing literature and thus strengthening the growing tendency verified by this study.
\end{abstract}

KEY-WORDS: Occupational therapy/trends. Health-disease process. Hospital care. Ocupational therapy department, hospital. Scientific publication indicators.

\title{
REFERÊNCIAS
}

BRASIL. INEP. Cadastro da educação superior. Disponível em: http://www.educacaosuperior.inep.gov.br/. Acesso em: 03 nov. 2008.

CAVALCANTI, A.; GALVÃO, C. (orgs.). Terapia ocupacional - fundamentação \& prática. Rio de Janeiro: Guanabara Koogan, 2007.

DE CARLO, M.; LUZO, M. C. (orgs.). Terapia ocupacional: reabilitação física e contextos hospitalares. São Paulo: Rocca, 2004.
GALHEIGO, S. M. Domínios e temáticas no campo das práticas hospitalares em terapia ocupacional: uma revisão da literatura brasileira de 1990 a 2006. Rev. Ter. Ocup. Univ. São Paulo, v.18, n. 3, 2007.

KUDO, A, et al. .(orgs). Fisioterapia, fonoaudiologia e terapia ocupacional em pediatria. 2a. ed. São Paulo: Sarvier, 1994.

SERVANTES, L.F. Terapia Ocupacional: pesquisa e atuação em oncologia. Campo Grande: UCDB, 2002. 